\title{
Für immer und ewig?
}

Einige - auch naturwissenschaftliche - Gedanken

Thomas Görnitz

Ewigkeit - was für ein großes und bedeutungsvolles Wort! Über die Jahrtausende war es an die religiösen Vorstellungen der jeweiligen Kulturen gebunden - und dies bleibt sogar dann noch so, wenn diese Vorstellungen ihre religiösen Konnotationen nicht mehr deutlich werden lassen.

Seitdem es den modernen Menschen auf der Erde gibt, gibt es die Vorstellung, dass mit dem Tod noch nicht alles vorbei sei. Nur der Mensch bestattet seine Toten, und die damit verbundenen $\mathrm{Ri}$ tuale zeigen, dass Vorstellungen über Ewigkeit untrennbar mit dem Menschsein verbunden sind. Soweit Überlieferungen zurückreichen, zeigen sich darin auch immer wieder Berichte über Verbindungen zwischen der Zeit und der Ewigkeit.

\section{Die Menschen suchen die Verbindung zur Ewigkeit}

$\mathrm{Zu}$ den ältesten überlieferten kulturellen Zeugnissen der Menschheit, also bereits aus der Steinzeit, gehören große Anlagen, die der Erfassung der Zeitabläufe gewidmet waren. Weiträumige Sonnenobservatorien, wie man sie bei Goseck an der Saale gefunden hat, zeugen von ihrer Ausdehnung her und auch im Hinblick auf ihr Umfeld, dass diese nicht nur der bloßen astronomischen Feststellung der Sonnenwende gedient haben. Noch deutlicher wird dies bei der bekannten Anlage von Stonehenge. Dort hatte man tonnenschwere Steine aus großer Entfernung herangeführt und damit eine Anlage errichtet, welche Jahrtausende überdauert.

Etwa in dieser Zeit baute man auch die ersten Pyramiden in Ägypten. Sie zeigen ebenfalls eine Ausrichtung nach den Himmelserscheinungen. Die Kanten der Pyramiden liegen sehr exakt in der Richtung, in der vor etwa viereinhalb Jahrtausenden der damalige Polarstern zu finden war. Diese riesigen Anlagen waren bekanntlich die Grabstätten der Pharaonen, die einbalsamiert wurden, damit sie wohlbehalten in eine transzendente Welt überwechseln konnten und somit nach ihrem irdischen Tod weiterhin für das Wohl ihres Volkes 
sorgen können. Herrscher in anderen Kulturen bekamen Sklaven und Frauen in großer Zahl mit in ihre Grabstätte, damit diese ihnen im Jenseits zurVerfügung stehen konnten.

Auch die Nebra-Scheibe, die aus der Bronzezeit vor etwa 4000 Jahren stammt, verdeutlicht das Bestreben der Menschen, das Geschehen am gestirnten Himmel fassbar zu machen und damit auch den Zeitablauf überhaupt. Einige der Symbole auf dieser Scheibe haben offenbar ebenfalls eine Beziehung zum Religiösen.

Natürlich wird es bei allen diesen Beobachtungen des Himmelsgeschehens auch um Aussaat- und Erntetermine gegangen sein. Aber deren Festlegung hätte man auch mit wesentlich geringerem Aufwand erreichen können. Die gewaltigen Anlagen lassen erkennen, dass die Menschen auch zur damaligen Zeit das Bestreben hatten, einen Kontakt zur Transzendenz zu begründen. Der Ablauf der Zeit und der mit dem Tode erfolgende Austritt aus der Zeit hat die Menschen immer tiefgreifend beschäftigt.

Der Kern der christlichen Botschaft besteht darin, dass mit Christus der Tod nicht mehr als das absolute Ende begriffen werden muss. Vor allem nachdem das Christentum zur staatstragenden Religion geworden war, wurde seine frohe Botschaft mit Vorstellungen über eine Hölle und ein der Läuterung dienendes Fegefeuer angereichert. Im Laufe der Geschichte finden sich dann die verschiedensten Ausmalungen dieser Ansichten. Im Mittelalter wurde beispielsweise der Petersdom in Rom durch die Zusage finanziert, dass dafür gespendete Gelder die Verweilzeit der Spender und der von ihnen damit Begünstigten im Fegefeuer verkürzen würden.

Bei Denkern wie Nicolaus von Cues und Giordano Bruno werden bereits in einer Verbindung von Wissenschaft und Religion moderne Gedanken auch über die Kosmologie vorbereitet. Isaac Newton, der mit der klassischen Mechanik die Physik als rechnende Wissenschaft etabliert hat, hatte den Raum als "Sensorium Gottes" interpretiert und wohl auch die Zeit im Zusammenhang damit gesehen. Wegen der Verbindung zum Göttlichen musste Newton diesen physikalischen Größen eine jeweils bis ins Unendliche reichende Ausdehnung zusprechen. Hierbei wurde die Ewigkeit des Zeitablaufes abgebildet auf den Zahlenstrahl, der von minus bis plus Unendlich reicht. 


\section{Die Verbannung der Transzendenz}

Spätestens seit der Aufklärung ist in der Gesellschaft auch im Allgemeinen eine Veränderung im Blick auf Zeit und Ewigkeit eingetreten. Im Gefolge der überaus erfolgreichen Naturwissenschaften wurden spirituelle und religiöse Vorstellungen immer mehr als Kinderglaube abgetan.Vor allem im Rahmen der Biologie gehört es zu den Standardvorstellungen, dass mit dem Tod eines Lebewesens außer dem verwesenden Körper nichts überbleibe.

Die Vorstellungen und ein Nachdenken über eine unendliche Zeitdauer - so wird der Begriff der "Ewigkeit" oftmals interpretiert gehören jedoch zum Kern des Wesens des Menschen. Dies wird auch daran sichtbar, dass die Idee der Ewigkeit mit der Aufklärung nicht etwa verschwunden ist, sondern dass sie vielmehr aus dem Bereich der Religionen teilweise in den Bereich des naturwissenschaftlichen Denkens hinübergewandert ist.

So galt es bis vor etwa 100 Jahren in der Naturwissenschaft als eine unbestreitbare Tatsache, dass der Kosmos seit einer unendlichen Zeit besteht und dass er noch für eine unendliche Zeit weiterbestehen würde. Aber auch später gab und noch heute gibt es viele Wissenschaftler, für welche die unendliche Dauer der Zeit und damit des Kosmos ein unbestreitbarer Glaubenssatz ist.

Bevor wir uns jedoch mit der Ewigkeit und einer unendlichen Zeitdauer befassen, ist es vielleicht sinnvoll, einen kurzen Blick auf das Verständnis der Zeit selbst zu werfen.

\section{Naturgesetze}

Das erste, woran man aus naturwissenschaftlicher Sicht bei einer Sentenz wie "immer und ewig" denken mag, ist die mit den $\mathrm{Na}-$ turwissenschaften fast immer verbundene Vorstellung, dass die $\mathrm{Na}-$ turgesetze für immer gelten sollen. Nun weiß man aber heute, dass der kosmische Raum expandiert. Daraus folgt, und darüber wird selten reflektiert, dass es niemals und nirgendwo zwei identische Situationen im Kosmos geben kann. Zu jedem beliebigen Zeitpunkt ist der Kosmos mit den Objekten und den Kräften in ihm anders als zu jedem anderen Zeitpunkt. Ein Gesetz macht jedoch nur Sinn für "Gleiches«. Wenn es jedoch nichts Gleiches gibt, was dann bitteschön ist der Sinn eines Gesetzes? 
In den mathematischen Naturwissenschaften hat man eine Teillösung zu diesem Problem dadurch gefunden, dass man zwischen Gesetz und Anfangsbedingungen unterscheidet.

Die Struktur einer Differenzialgleichung beschreibt zu einem gegebenen Kraftgesetz die Schar sämtlicher möglicher Bahnkurven. Damit wird es beispielsweise möglich, für einen Stern mit seiner fest vorgegebenen Masse und für einen fiktiven Planeten mit einer ebenfalls festen Masse jede mögliche Bahn des Planeten zu berechnen. In diesem Idealfall, in welchem man den gesamten restlichen Kosmos ignoriert, kann man diese Berechnungen sogar für beliebig lange Zeiträume genau durchführen. Mit anderen Worten: Dadurch, dass man den Rest der Wirklichkeit in diesem Fall sunter den Teppich kehrt‘, kann man diese Bewegung für eine unendlich lange Zeit prognostizieren.

Aber natürlich wissen wir alle, dass der Kosmos mehr ist als nur eine Sonne und ein Planet. Und seit Newton weiß man auch, dass die Gravitationswirkung der übrigen Sterne beliebig weit reicht. Sie wird zwar mit wachsender Entfernung immer kleiner, jedoch niemals zu null. Außerdem weiß man auch, dass schon bei drei Körpern Bewegungen möglich werden, bei welchen winzigste Einflüsse eine vollständige Veränderung der Bahnbewegung verursachen können. Das Schlagwort hierfür ist "deterministisches Chaos" - und chaotische Bewegungen lassen sich in der Regel nur für beschränkte Zeitintervalle vorherberechnen, - jedenfalls nicht für unendlich lange Zeiten wie bei einer Sonne und einem Planeten.

Wegen der Expansion des Kosmos verändern sich die wirkenden Kräfte ebenfalls ständig - und es gibt sogar mehr als nur drei Körper, was ein chaotisches Verhalten nicht unbedingt weniger wahrscheinlich werden lassen wird. Daher ist es stets notwendig abzuwägen, welche Anteile der Wirklichkeit ohne Schaden für die Beschreibung des jeweils anstehenden Problems ignoriert werden können und müssen.

Wenn wir von da aus noch einmal die Rolle der Naturgesetze reflektieren, so wird deutlich, dass sie Annäherungen an die Wirklichkeit sind, jedoch nicht die Wirklichkeit selbst. Natürlich erfassen die heute bekannten Gesetze die Abläufe in der Natur schon mit einer früher nicht vorstellbaren Genauigkeit. Dennoch müssen wir stets dafür offen sein, dass sich bei einer zunehmenden Genauigkeit unserer Untersuchungen neue Verhaltensweisen zeigen können, die mit den bisher bekannten Naturgesetzen noch nicht erfasst worden sind. 
Bei manchen Physikern gewinnt man den Eindruck, dass sich für sie eine ewige Gültigkeit der Naturgesetze zu einem absoluten Prinzip entwickelt hat, welches der Realität vorgängig ist und >über` der Natur steht. Danach würden die ewigen Naturgesetze festlegen, wie sich die veränderliche Realität dann gemäß ihrerVorgaben entwickelt. Diese These hat zwar den Rang eines sehr guten Arbeitsprinzips, sie sollte aber nicht wie eine geoffenbarte Wahrheit behandelt werden.

\section{Die Zeit der Uhren}

Die Einteilung der Zeit geschah schon immer über die natürlichen rhythmischen Vorgänge. Sie wurde festgemacht am Tag, am Monat und am Jahr. Diese wiederholen sich unablässig: "Solange die Erde steht, soll nicht aufhören Saat und Ernte, Frost und Hitze, Sommer und Winter, Tag und Nacht." (Genesis 8,21)

Diese »ewige Wiederkehr des Gleichen" hat auch in die philosophischen und religiösen Bilder über die Welt Eingang gefunden. Nach einem "Platonischen Jahr" - etwa 25.780 Jahre - sollte der Weltlauf wieder von vorn beginnen. Bei ihrer Rotation um die eigene Achse seiert die Erde ein wenig, so dass die Verlängerung der Erdachse einen Kreis am Himmel beschreibt. Gegenwärtig ist sie auf den Polarstern gerichtet. Das Platonische Jahr beschreibt die Dauer des Zeitraums, welcher notwendig ist, um die Erdachse wieder auf die gleiche Stelle am Himmel zeigen zu lassen.

Die Vorstellungen von einer Wiedergeburt auch in kürzeren Zeitabständen sind heute recht populär. Nach den Vorstellungen von mancher östlichen Religion wird mit der Wiedergeburt immer wieder ein neuer Lebenslauf eröffnet. Diese Bilder, die vor allem vom wiederkehrenden Jahreslauf inspiriert sind, beeinflussen die Menschen bis in die Neuzeit. So ist zum Beispiel nicht nur Nietzsches Philosophie der "ewigen Wiederkehr des Gleichen" davon stark geprägt.

\section{Der Ablauf der Zeit und ihre Messung}

Der zyklischen Zeiterfahrung, wie sie auch im Umlauf der Uhrzeiger sichtbar wird, steht eine andere - eine lineare - gegenüber. Ein Abreißkalender kann diese repräsentieren. 
Jeder erlebt an sich selbst, dass es zwar immer wieder Frühling wird, dass man sich selbst aber unwiderruflich verändert. Die Kinder wachsen heran, Enkel werden geboren, und in der Regel verlassen die eigenen Eltern und manchmal auch Freunde die Welt vor uns. Jedes Leben hat einen Anfang, einen einmaligen Ablauf und endet einmal. Die Einmaligkeit und die unwiederbringliche Gerichtetheit des Zeitablaufes unseres Lebens kehrt auch in religiösen Weltbildern wieder. Im jüdisch-christlichen Kulturkreis läuft die Zeit von einem Anfang, von der Schöpfung der Welt, zu ihrem Ende, dem »Jüngsten Tag«.

Für die Messgenauigkeit der modernen Naturwissenschaft ist das Zeitmaß, welches aus der Rotation der Erde abgeleitet wird, zu ungenau geworden. Heute wird die extreme Gleichförmigkeit von quantenphysikalischen Vorgängen verwendet, um Uhren mit einer früher unvorstellbaren Genauigkeit zu konstruieren. So liegt der Fehler, der für die Standards der Zeitmessung seit längerem erreicht wurde, unter 1 Sekunde im Laufe von etwa 20 Millionen Jahren. Damit kann man beispielsweise feststellen, dass die Erde im Winter etwas schneller rotiert als im Sommer. Jeder kennt von den Pirouetten der Eiskunstläufer den Vorgang, dass diese langsamer rotieren, wenn sie die Arme ausstrecken, und schneller, wenn sie diese heranziehen. Die Landverteilung auf der Erde ist so, dass die meisten Bäume, welche im Winter ihr Laub abwerfen, auf der Nordhalbkugel stehen - und der dadurch bewirkte Effekt auf die Erdrotation ist Dank ultragenauer Atomuhren messbar geworden.

\section{Relativität der Zeit?}

Wenn im Rahmen der Naturwissenschaften über Zeit gesprochen wird, dann wird dabei fast immer ein besonderer Wert auf Einsteins Relativitätstheorie gelegt. Über die Relativitätstheorie und die Zeit wird viel geschrieben und jeder Mensch erlebt an sich selbst, dass die Zeit in seinem subjektiven Zeiterleben keineswegs immer so verläuft, wie es von der Uhr angezeigt wird. Was kann man physikalisch dazu sagen?

Es ist eine zutreffende naturwissenschaftliche Aussage, dass in einem System die Zeit langsamer verläuft als bei uns, wenn dieses sich relativ zu uns schnell bewegt oder wenn es einer wesentlich stärkeren Beschleunigung ausgesetzt ist, als sie bei uns wirkt. Diese physikalische Aussage meint nicht, das dort »die Uhr anders läuft" als bei uns, sondern sie bedeutet, dass tatsächlich jeder beliebige Zeit- 
ablauf sich dort nach dieser Zeit richtet. Sämtliche physikalischen Vorgänge, Schwingungen und sonstige Bewegungen richten sich nach dieser Zeit, auch die physiologischen Abläufe, falls dort ein Lebewesen anwesend ist. Diese Relativität der Zeit in einem beschleunigten oder sich schnell bewegenden System ist natürlich erst einmal sehr verwirrend. Daher erhält man als Physikprofessor immer wieder einmal Briefe, in denen jemand seine Überzeugung kund tut, dass dies alles nicht wahr sein kann. Allerdings gibt es so viele experimentelle Befunde, welche ein solches Verhalten bestätigen, dass im Rahmen der Naturwissenschaft dagegen keine ernstzunehmenden Widerstände existieren.

Es sind nicht wenige Physiker, welche über die Subjektivität der Zeit schreiben. Manche von ihnen behaupten sogar, und dazu gehörte auch Einstein, dass die Zeit "eine Illusion" sei. Einige von diesen habe ich auf einer Tagung im Jahre 2002 in Bielefeld getroffen. Vielleicht kann man »das Illusionäre der Zeit« daran festmachen, dass der betreffende Tagungsband im Jahre 2014 erschienen ist. Aber ein wirklicher Beweis ist das wohl nicht.

Die Mehrheit der Teilnehmer jener Tagung befasste sich mit der Erkenntnis, dass es aus ihrer Sicht keinen Grund gibt, dass die Zeit nur in einer Richtung verläuft. Dazu muss man feststellen, dass es zutreffend ist, dass die mathematische Struktur vieler wichtiger Gleichungen in der Physik genau so ist, dass man für die von ihnen beschriebenen Zusammenhänge die Zeit problemlos vorwärts oder rückwärts laufen lassen kann. Dabei wird aber übersehen, dass es aus Gründen der simplen Einfachheit in der Physik so eingerichtet wird, dass die Gleichungen genau diese Struktur besitzen. Sie sind so einfacher zu behandeln - und wenn man sie auf ein reales Problem anwendet, dann weiß man sowieso, wie die wirkliche Zeit abläuft. Übrigens, wenn ich später einen dieser Teilnehmer wiedergetroffen habe, so war keiner von ihnen jünger geworden. Trotzdem befassen sich viele Physiker noch immer mit dem sogenannten "Problem des Zeitpfeils", also der Erkenntnis, dass - im Gegensatz zu vielen Gleichungen der Physik - alle Abläufe in der Natur gemäß der von uns erlebten normalen Zeit verlaufen.

Dabei wird eine einfache naturphilosophische Erkenntnis missachtet. Carl Friedrich von Weizsäcker hat in seinen naturphilosophischen Betrachtungen stets gezeigt, dass die Struktur der Zeit mit ihrer Unterscheidung zwischen Vergangenheit, Gegenwart und Zukunft eine Voraussetzung dafür ist, überhaupt sinnvoll eine empirische Naturwissenschaft betreiben zu können. Empirie bedeutet schließlich, Erfahrungen zu machen, um daraus zu lernen und um 
aufgrund der Erfahrungen Prognosen erstellen zu können. Damit ist natürlich bereits der Unterschied zwischen den drei Modi der Zeit vorausgesetzt worden. Wenn eine solche Unterscheidung zwischen Vergangenheit und Zukunft nicht möglich ist, dann verliert auch der Begriff der Empirie oder der Erfahrung seinen Sinn. Ein solcher Zugang zur Zeit, wie ihn Weizsäcker eröffnet hat, erlaubt ein sehr viel entspannteres und natürlicheres Verhältnis zu dieser und auch zu den Vorstellungen, die wir mit dem Begriff der Ewigkeit kennzeichnen.

Weizsäcker berichtete gern von einem Vortrag, den er im Jahre 1938 über den von ihm entdeckten Prozess der Energieerzeugung in der Sonne gehalten hatte. Dabei ließ er die Bemerkung einfließen, dass das damit mögliche Alter der Sonne gut zu dem Alter des Kosmos passen würde, welches aus der damals noch nicht lange bekannten Rotverschiebung der Galaxien abgeleitet werden könnte. Der im Kolloquium anwesende Nobelpreisträger Walter Nernst wurde daraufhin richtig zornig und erklärte, dass der Gedanke eines Alters des Universums keine Naturwissenschaft sei. Hier war also ein Glaubenssatz in Gefahr, nämlich der Glaube an ein Universum ohne Anfang und Ende, ein Glaube, der allerdings - und nicht nur unter Naturwissenschaftlern - nicht so selten ist. Bis heute erscheinen immer wieder einmal kosmologische Theorien, die entweder den Urknall umschiffen wollen oder welche über die Zeit davor - also über die Zeit vor der Existenz der Zeit - Überlegungen und Rechnungen anstellen.

$\mathrm{Zu}$ dieser Tradition gehörte auch Albert Einstein. Auch er meinte $\mathrm{zu}$ wissen, dass das Universum in der Zeit weder einen Anfang noch ein Ende hat. Er musste jedoch erkennen, dass aus seinen Gleichungen der Allgemeinen Relativitätstheorie kein Weltmodell mit einer unendlichen zeitlichen Dauer folgen würde. Da er keinen zeitlichen Beginn der kosmischen Entwicklung wünschte, hatte er schließlich seine Gleichungen solange verändert, bis sie seiner Hypothese einer unendlichen Dauer ohne einen Anfang genügten. Er hatte dazu die so genannte kosmologische Konstante postuliert, die bis heute in der Relativitätstheorie ziemliches Kopfzerbrechen bereitet.

\section{Kosmische Zeit}

Alexander Alexandrowitsch Friedmann hatte 1922 als erster zeitlich veränderliche Weltmodelle publiziert. Der belgische Theologe, Mathematiker und Physiker Georges Lemaitre hatte im Rahmen 
der Allgemeinen Relativitätstheorie 1927 ein Weltmodell berechnet, welches mit einem sehr dichten Anfangszustand begann. Er sprach vom »Uratom", heute nennt man es "Big Bang".

Mit den bereits sehr genau gewordenen Beobachtungen des Kosmos, die heute zur Verfügung stehen, zeigt es sich, dass Modelle von diesem Typ sehr gut zu den Daten passen. Der Kosmos ist im Großen von einer erstaunlichen Gleichförmigkeit, so wie es in diesen Weltmodellen aus Gründen der Einfachheit angenommen wird. Die Gesamtheit der Daten verweist darauf, dass vor etwa 13,8 Milliarden Jahren der Kosmos sehr viel dichter und heißer war als heute und sich in einem singulären Zustand befunden hat. Dieser war von allem unterschieden, was wir heute an wissenschaftlichen Vorstellungen haben. Das Bild vom Urknall, von einem Beginn derjenigen Realität, die wir naturwissenschaftlich untersuchen können, die also in Raum und Zeit existiert, ist offenbar nicht zu widerlegen.

Einstein hielt solche Lösungen mit einem sich verändernden kosmischen Raum lediglich für Mathematik - ohne physikalische Bedeutung. Es ist ein gewisser Witz der Wissenschaftsgeschichte, dass 1929 die Fluchtbewegung der Galaxien von Edwin Hubble publik gemacht wurde. Dies wurde bald als Hinweis auf die Expansion des Kosmos verstanden. Damit waren Einsteins Überlegungen hinfällig, die zu seiner Abänderung der Allgemeinen Relativitätstheorie durch die Einführung der sogenannten kosmologischen Konstante geführt hatten.

Lange Zeit hatte man dann die kosmologischen Modelle ohne diesen Term berechnet. Im Ergebnis erhielt man zumeist Modelle, welche einen zeitlichen Beginn und ein zeitliches Ende besitzen. Dabei begann der Kosmos mit einer Singularität, dann dehnte sich der Raum aus, um alsdann, nach dem Erreichen eines Maximums, wieder zu einer Singularität zusammenzuschrumpfen. Diese Modelle spiegelten in meiner Studenten- und Doktorandenzeit den Typus von Weltmodell wider, von dem alle Fachleute 'wussten', dass er zutreffend ist. Als ich vor 30 Jahren ein Modell vorstellte, bei dem der Ortsraum immer weiter expandiert und sich nicht wieder in einen Endknall zu einem Punkt zusammenzog - was sehr gut zu den heutigen sehr viel besseren Beobachtungsdaten als damals passt -, wurde die Arbeit erst einmal abgelehnt.

Um dem offenbar im Menschen fest verwurzelten Wunsch nach "Ewigkeit" Genüge zu tun, wurden die damaligen wieder kontrahierenden Modelle dann zumeist vor dem Urknall und nach dem Endknall in gleicher Weise fortgesetzt. Sie entsprachen damit sehr genau Nietzsches »Wiederkehr des ewig Gleichen«. 
Heute wird die kosmologische Konstante unter dem Namen "Dunkle Energie« wieder verwendet, weil in den bisher üblicherweise angewandten Modellen ohne sie die Abweichungen von den Beobachtungsdaten zu groß werden würden. Die Schwierigkeiten mit dieser unverstandenen Konstante zeigen sich auch darin, dass eine quantenfeldtheoretische Berechnung für diese einen Wert liefert, der im Vergleich zu den Beobachtungen etwa um den Faktor $10^{120} \mathrm{zu}$ groß ist (das ist eine Eins mit 120 Nullen).

Auch mit der Dunklen Energie ist noch immer eine Differenz zwischen der Beobachtung und den Modellen gegeben. So hat man dazu noch die "Inflation" erfunden. Dieser Zaubertrick soll den Kosmos am Anfang so schnell aufblasen, dass damit die heute beobachtbare Homogenität der Hintergrundstrahlung erklärbar werden soll. Alles, was heute beobachtbar ist, wäre demnach ein so winziger Ausschnitt aus dem postulierten Gesamtkosmos, dass dieses Bruchstück zu klein für wahrnehmbare Schwankungen oder Differenzierungen wäre und somit so glatt erscheinen müsste, wie wir es heute in den astronomischen Beobachtungen sehen. Der riesige Rest hätte sich demnach so weit wegbewegt, dass das Licht von dort keine Möglichkeit hat, in den 13,8 Milliarden Jahren seit dem Urknall bis zu uns zu gelangen. Und da dieser "Rest « - also fast alles - immer weiter wegfliegt, wird uns nach dem Erklärungsversuch der Inflation das Licht von dort niemals erreichen. Dass mit dieser wilden Annahme die gesamte beobachtende Kosmologie auf einen bedeutungslos winzigen Bruchteil der vorgeblichen Wirklichkeit eingeschränkt wird, wird im Zusammenhang mit der Inflation nicht reflektiert.

\section{Universelle Zeit}

Wir haben hier relativ ausführlich über den Kosmos und seine zeitliche Entwicklung gesprochen, da darüber weithin große Unklarheiten existieren.

So haben die sehr guten astronomischen Beobachtungen und astrophysikalischen Einsichten zur Hintergrundstrahlung gezeigt, dass es im Widerspruch zu manchen Thesen über die Relativitätstheorie im gesamten Kosmos durchaus ein ausgezeichnetes Bezugssystem gibt. Damit wiederum gibt es auch eine ausgezeichnete kosmische Zeit. Diese kosmische Zeit darf so, wie es auch von Newton gesehen wurde, als universell begriffen werden. Im Unterschied zu Newton wissen wir jedoch heute, dass in schnell bewegten Systemen, die des- 
wegen auch in gewisser Weise vom übrigen Kosmos isoliert sind, die Zeit langsamer verläuft als diese universelle kosmische Zeit. "Schnell bewegt" bedeutet, dass sie mit ihrer Geschwindigkeit relativ zum Kosmos einen ziemlich großen Prozentsatz der Lichtgeschwindigkeit von $300.000 \mathrm{~km} / \mathrm{s}$ erreichen.

Da sich die Erde lediglich mit 500 km/s im kosmischen Raum bewegt, ist der Zeitablauf im Kosmos insgesamt und der auf der Erde im Wesentlichen identisch. Wenn man allerdings genötigt ist, sehr genau zur arbeiten, dann können die zeitlichen Effekte von Relativitätstheorie und Quantentheorie nicht mehr ignoriert werden. Dies ist beispielsweise bei den Satelliten der Fall, welche die Signale für die GPS-Empfänger aussenden. Dort fliegen sehr genaue Atomuhren mit, die eine exakte Zeitbestimmung ermöglichen. Ohne diese könnte die mögliche Ortsgenauigkeit, die aus deren Signalen errechnet wird, nicht erreicht werden.

\section{Quantentheorie}

Sehr viel interessanter und sehr viel weniger verständlich dargestellt wird das zeitliche Verhalten in Systemen, die mit der Quantentheorie beschrieben werden müssen.

Die Quantentheorie ist der Bereich der Physik, der immer erst dann angewendet werden muss, wenn die Beschreibung der Natur sehr genau werden soll oder genau werden muss, damit die Beschreibung nicht in einem zu großen Widerspruch zur Erfahrung steht.

Die Quantentheorie ist eine Theorie über Ganzheiten und über Möglichkeiten. Sie basiert auf zwei Grundprinzipien, die für uns Menschen vollkommen evident sind:

1. Ein Ganzes ist oftmals wesentlich mehr als nur die Summe der Teile, in die man es zerlegen könnte oder aus denen es aufgebaut wurde.

2. Nicht nur Fakten, sondern auch Möglichkeiten beeinflussen ein Geschehen.

Diese nahezu trivial erscheinenden Thesen müssen in eine klare mathematische Struktur übersetzt werden - und genau diese Struktur ist die Quantentheorie. Die Konsequenzen, die sich aus diesen Offensichtlichkeiten ergeben, sind jedoch keineswegs so trivial und so alltäglich, wie diese beiden Sätze es vermuten lassen würden.

Die Quantentheorie hat uns Menschen gezeigt, dass in der Tiefe der Erscheinungen viele Unterschiede relativiert werden, die im 
Alltag unüberbrückbar erscheinen. Oftmals noch findet man Menschen, die an Lenins Leitsatz glauben: „Es gibt nur die Materie, und die Bewegung ist ihre Grundeigenschaft«. In der Wissenschaft jedoch weiß man seit langem, dass Materie und Bewegung ineinander umgewandelt werden können. Allerdings verwendet man in diesen Zusammenhängen den physikalischen Begriff der "Energie « anstelle von "Bewegung". Das gleiche gilt für die Quanten von denjenigen Erscheinungen, die als Stoff und als Kraft bezeichnet werden. Die Quantentheorie relativiert die Unterscheidung von Lokalisiertheit und Ausgedehntheit. Sie zeigt uns, dass eine Erscheinung, welche in einem Kontext wie eine Eigenschaft begriffen werden kann - zum Beispiel "Bewegung « - in einem anderen Kontext als eigenständiges Objekt verstanden werden muss -, so sind Lichtquanten, die Photonen, Objekte von reiner Energie.

\section{Wirklich einfache Strukturen}

Schließlich zeigt es sich, dass der bisher in der Physik verfolgte Weg, das Einfachere in immer kleineren Teilchen zu suchen, zwar bis zur Chemie überaus erfolgreich ist, jedoch von dort aus weiter ins immer Kleinere in eine tiefe Sackgasse führt. Aus der mathematischen Struktur der Quantentheorie folgt, dass die einfachsten Strukturen keineswegs "kleinste materielle Teilchen" sein können, sondern dass es Strukturen sind, die heute als Quantenbits bezeichnet werden.

Carl Friedrich von Weizsäcker war der erste, der davon gesprochen hat, dass die tatsächlich einfachsten Quantenstrukturen die Quantenbits sind. Er war durch Überlegungen aus der Logik zu diesen Einsichten gekommen und nannte diese allereinfachsten Quantensysteme "Ur-Alternativen". Da sie bei ihm jedoch noch immer mit Wissen und mit Bedeutung verknüpft waren, war es notwendig, noch abstrakter zu werden, als es Weizsäcker bereits war. Schließlich hat Bedeutung stets einen großen subjektiven Anteil und kann daher nicht von einer Wissenschaft wie der Physik erfasst werden, die auf das Objektive zielt.

Um den gedanklichen Kurzschluss von Informationen als Bedeutung zu vermeiden, wurde für diese abstrakte und bedeutungsfreie Quanteninformation ein Begriff geprägt, der keinerlei Vorstellungen und damit auch keine falschen hervorrufen kann: "Protyposis"

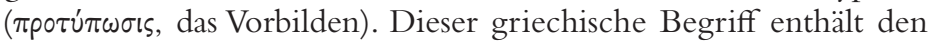
Wortstamm "typeo - ich präge ein", der beispielsweise auch im "Archetypus« vorkommt. 
Diese abstrakten bedeutungsfreien Quantenbits sind die tatsächlich grundlegenden und einfachsten Quantenstrukturen, aus denen die ungeheure Fülle der Erscheinungen hergeleitet werden kann.

Mit der Protyposis wird das Ziel der Naturwissenschaften erreicht, all die komplizierten Objekte in der Natur aus tatsächlich einfachen Strukturen aufzubauen. In umfangreichen quantentheoretischen Rechnungen wurde gezeigt, dass Quantenteilchen aus einer unbegrenzten Anzahl von Quantenbits aufgebaut werden können - so wie ein Quantenfeld wiederum aus einer unbegrenzten Anzahl von Quantenteilchen aufgebaut werden kann. Auf diesem Weg wird es möglich zu verstehen, wie aus Einfachem etwas Kompliziertes und Neues entstehen kann, auch solche physikalischen Phänomene, die bisher als "Dunkle Energie» und »Dunkle Materie» bezeichnet werden oder diejenigen, für welche man die "Inflation« erfunden hatte.

Und schließlich folgt mit der Protyposis, dass unsere Gedanken lediglich andere Erscheinungsformen dieser Grundsubstanz sind als die Atome in unserem Gehirn, aber dass sie im Wesenskern nicht von diesen verschiedenen sind.

Mit der Protyposis wird somit ein vollkommen neues Verständnis von Materie eingeführt. Materie erweist sich als geformte, kondensierte und dichtgepackte Quanteninformation. Und das Bewusstsein kann definiert werden als Quanteninformation, die sich selbst erleben und kennen kann.

Quanteneigenschaften werden an einem System erst dann deutlich bemerkbar, wenn es isoliert ist. Wenn jedoch ein System so gut von seiner Umwelt isoliert ist, dass an ihm Quanteneigenschaften erkennbar werden können, dann zeigt es sich, dass innerhalb dieses Systems kein Zeitablauf mehr existiert. Niels Bohr prägte dafür den Ausdruck »individueller Prozess". Die Quantentheorie ist also eine Theorie über Möglichkeiten, in ihrer theoretischen Struktur ist kein Platz für Fakten.

\section{Möglichkeiten und Fakten}

Ein Quantensystem, solange es isoliert vom Rest der Welt verbleibt, befindet sich daher in einer ausgedehnten Gegenwart. Dies klingt zwar extrem merkwürdig, aber erst durch das Konstatieren von Fakten lassen sich Zeiten in die Bereiche von "vor einem Ereignis" und "nach einem Ereignis" untergliedern. Die erste Darstellung dieses Zusammenhangs stammt von Weizsäcker. Viele Jahre später hat dann Archibald Wheeler mit großer publizistischer Wirkung von 
"verzögerter Wahl" (»delayed choice«) gesprochen. Dies wurde oft so missverstanden, als ob man im Rahmen der Quantentheorie nachträglich eine Vergangenheit wählen könnte.

Den zeitlichen Vorgang an einem Quantensystem sollte man am einfachsten so erklären, dass man am Ende eines individuellen Prozesses natürlich festlegen kann, in welcher Weise man dasVergangene zwischen Beginn und Ende des individuellen Prozesses ebenfalls als eine Reihe von fiktiven - also fantasierten - Fakten beschreiben will. Allerdings macht die Quantentheorie im Grunde genommen deutlich, dass es zwischen Anfang und Ende des individuellen Prozesses am betreffenden System gar keine Fakten gegeben hat.

Aus dem Fächer von Möglichkeiten eines Quantensystems bleibt nach dem "Messprozess" eine Möglichkeit als Faktum vorhanden. Die Information über die anderen möglichen Zustände ist aus dem System entwichen.

Damit an einem Quantensystem tatsächlich ein Faktum kreiert worden ist, ist es notwendig, dass die abgestrahlte Information auch unter sämtlichen theoretischen Gesichtspunkten niemals die Chance hat, jemals in dieses System zurückzugelangen. An dem sogenannten Quantenradierer hat man lernen können, dass ein solcher unwiederbringlicher Verlust von Informationen über Quantenmöglichkeiten der entscheidende Vorgang ist, um ein Faktum entstehen zu lassen. Die Information muss also in die Tiefe eines expandierenden Kosmos entschwinden. Nur damit ist gesichert, dass eine Rückkehr auch theoretisch unmöglich ist. Diese Verbindung der Quantentheorie zur Kosmologie lässt etwas von dem universellen Zusammenhang aller Naturerscheinungen deutlich werden.

Das Aufheben der Isolierung und das damit bewirkte Setzen eines Faktums bezeichnet man in der Physik als Messvorgang. Dabei wird der normale Ablauf unterbrochen, also die zeitliche Veränderung von Möglichkeiten innerhalb eines Quantensystems. Wird dann die Isolierung wiederhergestellt, dann kann sich aus diesem Faktum wieder eine neue Fülle von quantischen Möglichkeiten entwickeln.

\section{Augustinus und die Quantentheorie}

Die Zeitfreiheit innerhalb eines Quantensystems öffnet den Blick auf einen Zusammenhang, der eng mit unserem Thema verbunden ist. 
Was passiert, wenn eine Gegenwart nicht durch Fakten in Vergangenheit verwandelt werden würde? Dazu schreibt Augustinus: "Wenn dagegen die Gegenwart immer gegenwärtig wäre und nicht in die Vergangenheit übergänge, so wäre sie nicht mehr Zeit, sondern Ewigkeit. « Eine solche andauernde Gegenwart ist jedoch das, was ein Quantensystem gerade auszeichnet.

Erst durch einen Verlust von Information über alle möglichen Quantenzustände bis auf einen von diesen kann an einem solchen System ein Fakt, eine Tatsache, konstituiert werden. Und erst mit Fakten wird - wir wiederholen uns - eine zeitliche Unterteilung im Sinne von "vor einem Fakt" und "nach einem Fakt" möglich. Erst dies erlaubt die Gliederung der Zeit in Vergangenheit, Gegenwart und Zukunft.

Wenn wir die Feststellung von Augustinus noch einmal überdenken, dann erkennen wir, dass wir für eine solche unzergliederte Gegenwart normalerweise einen anderen Begriff als "Ewigkeit» verwenden. Wir sprechen von einem "Augenblick«.

Wenn wir unsere Gedanken und Gefühle naturwissenschaftlich einordnen wollen, so können wir sie als bedeutungsvolle Quanteninformation bezeichnen. Unsere Gedanken, aber nicht unser Gehirn als Ganzes können wir damit als eine Quantenstruktur begreifen, die so gut von der Umwelt isoliert ist, dass sie von außen lediglich ungefähr erkennbar ist.

Können wir etwas von dieser "Zeitfreiheit« spüren?

In derVersenkung, in der Meditation ist dies für manche erfahrbar. Aus naturwissenschaftlicher Sicht kann man sagen, dass besonders in der Meditation ein Vorgang stattfindet, der als ein Bohrscher individueller Prozess verstanden werden kann. In solchen Zuständen werden Erlebnisse möglich, die eine Erfahrung einer ausgedehnten zeitfreien Gegenwart ermöglichen. Damit kann uns Menschen bereits hier etwas von dem vermittelt werden, was nach der Definition von Augustinus als Ewigkeit bezeichnet werden darf.

Diese Vorstellung von Ewigkeit zeigt noch etwas anderes auf als die gemeinhin verbreiteten Bilder von Ewigkeit als einer unendlichen Abfolge von Fakten.

\footnotetext{
1 Augustinus, Bekenntnisse, eingeleitet und übertragen von Wilhelm Thimme, Zürich 1950, Elftes Buch, XIV, 312.
} 


\section{Wie kann man es heute sehen?}

Wir Menschen finden uns in einem Kosmos vor, dessen Grundstrukturen, die Quantenbits der Protyposis, durch ihre ständige Zunahme die Expansion dieses Kosmos bewirken. Seit ihrem unstrukturierten Anfang hat ihre heute riesige Anzahl die Fülle von Erscheinungen ermöglicht, die sich in der kosmischen und biologischen Evolution entwickelt haben. Mit der Protyposis können auch der Körper und das Bewusstsein als verschiedene Ausformungen dieser Substanz begriffen werden, welche in der Tiefe eher mit dem Ähnlichkeiten hat, was man als etwas Geistiges bezeichnen würde und weniger mit den üblichen Vorstellungen von etwas Materiellem.

Von hier und jetzt entweicht ständig Information in die Tiefe des expandierenden kosmischen Raumes, so dass wir durch das fortwährende Entstehen von Fakten einen Zeitablauf wahrnehmen, in welchen wir eingebettet sind.

Wenn Ewigkeit mit Augustinus auch so interpretiert werden kann, dass in ihr keine Fakten entstehen, dann kann der Spruch aus dem Neuen Testament: »Bei Gott ist kein Ding unmöglich« (Lk 1,37) mit einer neuen Interpretation verbunden werden: "Im letzten Grund verbleibt alles im Bereich des Möglichen."

Dies passt zur Struktur der Quantentheorie, denn für den Kosmos als Ganzen kann nichts »in die Tiefe des kosmischen Raumes entschwinden«. Dies würde bedeuten, dass es Fakten lediglich für lokalisierte Systeme gibt, zum Beispiel für Menschen auf der winzigen Erde in dem riesigen Kosmos.

Solange wir leben, ist unser Bewusstsein an ein lebendiges Gehirn als Träger gebunden. Diese Trägerschaft und die damit verbundene Lokalisierung wird im Tode aufgelöst. Ohne die Bindung an einen materiellen oder energetischen Träger ist diejenige Form der Protyposis, die wir im Leben als unsere Psyche bezeichnen können, weder hier noch jetzt, sie ist dann gleichsam immer und überall. Allerdings erlaubt eine solche Feststellung keinerlei Aussage über eine noch mögliche Struktur solcher Quanteninformation. Kann es dann noch etwas geben, was mit unserer früheren Existenz verbunden ist? Unsere naturwissenschaftlichen Kenntnisse kommen damit an ein Ende - sie ermöglichen aber ein Nachdenken über Beziehungen zu manchen religiösen und spirituellen Erfahrungen.

Eine dazu passende Metapher, die auch berücksichtigt, dass die Grundlage der Wirklichkeit mit der Protyposis gegeben ist und die deren Beziehung zu unserem Bewusstsein aufgreift, könnte wie folgt formuliert werden: 
"Die Realität, das sind die möglichen Gedanken Gottes."

Auf jeden Fall kann damit ein Anstoß gegeben werden, einerseits allzu simple Vorstellungen über »immer und ewig" neu zu überdenken. Andererseits kann man erkennen, dass der Graben zwischen religiösen und wissenschaftlichen Vorstellungen und Bildern, der mit dem Beginn der Neuzeit aufgerissen wurde, weniger unüberbrückbar ist, als es scheint.

— Thomas Görnitz war bis zu seiner Pensionierung Professor für Didaktik der Physik an der Goethe Universität Frankfurt. Schwerpunkt seiner Forschung ist die mathematische Struktur der Naturwissenschaft, speziell der Quantentheorie sowie deren philosophische Durchdringung und verständliche Darstellung mit Schwerpunkten Kosmos, Leben, Bewusstsein. 